\title{
Homogeneidade espacial da precipitação pluvial na bacia hidrográfica do Rio Vacacaí, RS
}

\author{
Spatial homogeneity of pluvial precipitation in Vacacaí river watershed, RS, Brazil
}

\author{
Galileo Adeli Buriol ${ }^{\mathrm{I}}$ Valduíno Stefanel ${ }^{\mathrm{II}}$ Alexandre Swarowsky ${ }^{\mathrm{II}^{*}}$ \\ Rafael Tatsch de Oliveira Cademartori ${ }^{\mathrm{II}}$
}

\section{RESUMO}

\begin{abstract}
O objetivo, neste trabalho, foi determinar o número mínimo de anos com observações necessários para a obtenção de valores médios dos totais mensais de precipitação pluvial consistentes e a área de abrangência das estações pluviométricas, localizadas na bacia hidrográfica do Rio Vacacaí, estado do Rio Grande do Sul. Foram utilizadas as séries históricas de dados dos totais mensais de 13 estações pluviométricas localizadas na área e no entorno da referida bacia hidrográfica. O número mínimo de anos foi calculado pela relação entre o número de anos com observações e a estabilidade dos coeficientes de determinação. A abrangência das estações foi determinada pela relação dos coeficientes de determinação dos totais mensais de precipitação pluvial entre cada estação e a estação base, no caso Santa Maria, $e$ as distâncias lineares entre a estação base e essas estações. A partir dessas equações, foram determinadas as distâncias entre estações em que há $80 \%, 85 \%$, 90\%, 95\% e 99\% de concordância dos valores. Constatou-se que, para ter uma razoável precisão nos totais mensais de precipitação pluvial na área da bacia hidrográfica do Rio Vacacaí, são necessários em torno de 30 a 40 anos de observação e que, nos meses de dezembro, janeiro e fevereiro, a abrangência das estações pluviométricas é menor do que $1,0 \mathrm{~km}$ e nos outros meses uma concordância de $99 \%$ é obtida para distâncias de 1,3 a 2,2km, de 95\% para distâncias de 6,3 a $11,1 \mathrm{~km}$ e de $90 \%$ para distâncias de 12,5 a 22,2km.
\end{abstract}

Palavras-chave: série histórica, chuva, consistência.

\section{ABSTRACT}

The objective of this research was to determine the minimum number of years with observations needed to obtain the medium number of total monthly consistent precipitation values and the area of coverage of the pluviometric station located in the Vacacaí river watershed, in the Rio Grande do Sul state,
Brazil. It were used the historic series of precipitation data of 13 pluviometric stations located in the area and around the watershed. The minimum number of years was calculated from the relation between the number of years with observations and the stability of coefficients of determination. The station's area of coverage was determined from the relation between the coefficients of determination of the total monthly precipitation between each station and the base station (Santa Maria), and the linear distances between the base station and each station. From those equations, the distance between stations in which 80\%, 85\%, 90\%, 95\% e 99\% of the values has concordance was determined. It was found that, to have a reasonable precision in the total monthly precipitation in the Vacacaí river watershed, it is necessary between 30 and 40 years of observation and, in December, January and February months the coverage of the pluviometric stations is less than $1.0 \mathrm{~km}$ and on the other months to achieve a precision of $99 \%$ distances between 1.3 and $2.2 \mathrm{~km}, 95 \%$ distances between 6.3 and $11.1 \mathrm{~km}$ and $90 \%$ for distances between 12.5 and $22.5 \mathrm{~km}$.

Keywords: historical series, rain, consistence.

\section{INTRODUÇÃO}

Dentre os elementos climáticos, a precipitação pluvial é um dos que possui maior variabilidade temporal e espacial e um dos mais importantes no crescimento e desenvolvimento vegetal e, ainda, um dos que maior influência exerce nas atividades humanas. Sua importância pode ser expressa, por exemplo, na produção de biomassa vegetal, no abastecimento de água para a irrigação, energia e consumo humano e animal; e nos danos ambientais por inundações, erosão e seca.

ICurso de Engenharia Ambiental e Sanitária, Centro Universitário Franciscano (UNIFRA), Santa Maria, RS, Brasil.

IIUNIFRA, 97105-900, Santa Maria, RS, Brasil. E-mail: alexandre@unifra.br. *Autor para correspondência. 
Para não incorrer em interpretações errôneas nos resultados de parâmetros como a média, variabilidade, intensidade e tempo de retorno, é recomendado que todas as séries históricas de dados de precipitação pluvial sejam precedidas da determinação de sua consistência (CASTILLOS \& SENTIS, 1996; CAMARGO et al., 2005). A homogeneidade temporal das séries de dados pode ser realizada por métodos como o das Duplas Massas (NORDENSON, 1971; OMETTO, 1981; CASTILLOS \& SENTIS, 1996; BERTONI \& TUCCI, 2002), do cociente (CASTILLOS \& SENTIS, 1996) e o teste de Iterações para uma Amostra (SIEGEL, 1975). Entretanto, em função, principalmente, das variações topográficas, dos movimentos convectivos na atmosfera e da época do ano, a precipitação pluvial pode variar significativamente de um local para outro, mesmo estes estando próximos (MELLAART, 1999; CAMARGO et al., 2005). Assim, é importante determinar também a sua homogeneidade espaçotemporal, caso contrário, pode-se incorrer em erros ao determinar sua distribuição geográfica (CAMARGO \& HUBBARD, 1999; CAMARGO et al., 2005).

A bacia hidrográfica do Rio Vacacaí está localizada entre as províncias geomorfológicas da Depressão Central e do Escudo Sul-riograndense, estado do Rio Grande do Sul (RS, 1989; SEMA, 2009). Sua área é de, aproximadamente, $10.000 \mathrm{~km}^{2}$ (SEMA, 2009), constituindo-se numa das mais extensas bacias hidrográficas da região Central do estado. A sua importância está vinculada, principalmente, à utilização de suas terras e águas na agricultura e pecuária extensiva (PRÓ-GUAÍBA, 2011). A altitude nas nascentes do Rio Vacacaí, na

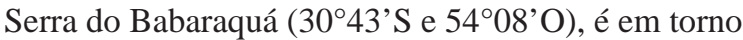
de 400m e, na sua foz, no Rio Jacuí, em média, de 20m (SEMA, 2009). Infere-se, assim, que pode haver diferenças importantes de precipitação pluvial entre os diferentes locais na área da bacia hidrográfica. Já foi constatado que as séries históricas de dados das estações pluviométricas localizadas na área e entorno da bacia hidrográfica do Rio Vacacaí são homogêneas (SILVEIRA et al., 2012). Entretanto, mesmo as séries temporais sendo consistentes, não é seguro utilizálas em estudo de sua variação espacial. Nesse caso, é importante determinar também a homogeneidade espaço-temporal, caso contrário, pode-se incorrer em risco de cometer erros no traçado das isoietas.

Neste trabalho, teve-se como objetivo determinar o número mínimo de anos com observações necessários para a obtenção de valores médios dos totais mensais consistentes de precipitação pluvial e a área de abrangência das estações pluviométricas, localizadas no perímetro e no entorno da bacia hidrográfica do Rio Vacacaí, estado do Rio Grande do Sul.

\section{MATERIAL E MÉTODOS}

Foram utilizados os dados dos totais mensais de precipitação pluvial de 13 estações pluviométricas, localizadas na área e no entorno da bacia hidrográfica do Rio Vacacaí - RS, todas com mais de 20 anos de observação, figura 1 . As séries históricas de dados foram copiadas dos arquivos do Departamento Estadual de Portos, Rios e Canais (DEPREC), atual Secretaria Estadual de Transporte e Hidrovias (SETH), estações de Caranguejo, Formigueiro, Passo das Tropas, Pau Fincado, São José da Porteirinha, São Marcos e São Sepé; do $8^{\circ}$ Distrito de Meteorologia (8o DISME), pertencente ao Instituto Nacional de Meteorologia (INMET), estações de Caçapava do Sul, Cachoeira do Sul e Santa Maria; da Fundação Estadual de Pesquisa Agropecuária (FEPAGRO), estações de Boca do Monte e São Gabriel, todas com sede em Porto Alegre; e do site Hidroweb do Departamento Nacional de Águas e Energia Elétrica (DNAEE), atual Agência Nacional das Águas (ANA, 2010), estação de Barro Vermelho.

A carta da bacia hidrográfica foi construída utilizando-se as Cartas Topográficas do Exército, da Diretoria de Serviço Geográfico (DSG) da $1^{\text {a }}$ Divisão de Levantamento (1ª̊DL), na escala 1:25.000. Utilizou-se a projeção UTM (Universal Transversa de Mercator), com predominância na zona 22, meridiano central 57\%, Datum horizontal SAD-69 (South American Datum 69), que possibilitou representar as áreas e distâncias. A rede de drenagem foi editada em ambiente virtual pelo uso do software ArcView 9.2 e, na elaboração do mapa planialtimétrico, foi empregada a imagem de radar do Shuttle Radar Topography Mission (SRTM), do sítio da Embrapa, cena com nomenclatura SH-22-V-D.

O número mínimo de anos com observações necessários para a obtenção de valores médios dos totais mensais consistentes das séries históricas dos dados das estações pluviométricas localizadas na área e no entorno da bacia hidrográfica do Rio Vacacaí foi obtido pela relação entre o número de anos de observações e a estabilidade dos coeficientes de determinação (CAMARGO et al., 2005). Para isso, foram utilizados os totais mensais das estações pluviométricas de Santa Maria (8o DISME), período de observação de 1912 a 2010, de Cachoeira do Sul (8o DISME), período de observação de 1912 a 1974, e de São Gabriel (FEPAGRO), período de observação 


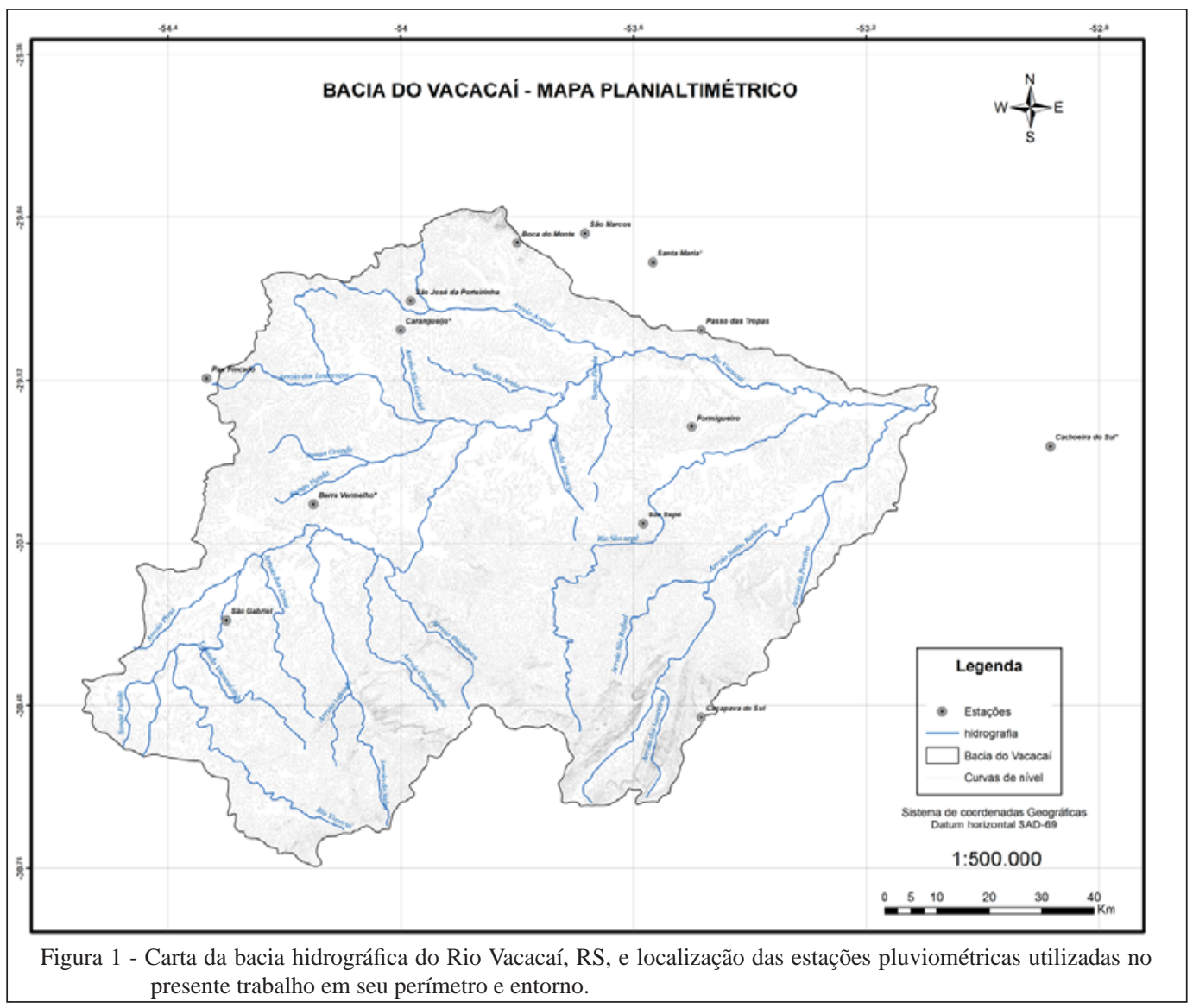

de 1965 a 2008. Para isso, foram calculados os coeficientes de determinação $\left(\mathrm{R}^{2}\right)$, para cada mês, com $10,11,12,13,14, \ldots, n$ anos de observações. Utilizouse, como base, a estação de Santa Maria, pertencente ao 8 DISME, tendo em vista que foi comprovado que a série de dados pluviométricos dessa estação é consistente (BURIOL et al., 2007). Foram traçados diagramas de dispersão, tendo nas abcissas o número de anos de observação e nas ordenadas os valores dos coeficientes de determinação $\left(\mathrm{R}^{2}\right)$.

A determinação da abrangência das estações pluviométricas foi realizada calculando-se, primeiramente, para cada mês, as correlações entre os totais mensais da estação base, no caso Santa Maria, e cada uma das outras estações consideradas. A associação da precipitação pluvial foi obtida pelo valor do coeficiente de determinação $\left(\mathrm{R}^{2}\right)$ e a variabilidade deste pelo erro padrão de estimativa (SEE). Esses valores foram relacionados com as respectivas distâncias lineares entre a estação pluviométrica base e as outras estações consideradas. As distâncias foram determinadas a partir das coordenadas geográficas de cada estação. Os valores de $\mathrm{R}^{2}$ são adimensionais e aqueles de SEE têm a mesma unidade do elemento climático, no caso da precipitação pluvial, em mm (CAMARGO et al., 2005).

Foram calculadas equações de regressão considerando $\mathrm{X}$ a distância em $\mathrm{km}$ de cada estação a Santa Maria e Y os $\mathrm{R}^{2}$ obtidos. A partir dessa equação, foram determinadas as distâncias entre estações em que há 80\%, 85\%, 90\%, 95\% e 99\% de concordância dos valores. Uma concordância de $90 \%$, por exemplo, indica que os totais de precipitação concordam em $90 \%$, ou seja, a variabilidade conjunta dos totais de precipitação das duas estações é de $90 \%$. Para isso, substituiu-se, nas equações, o valor de $\mathrm{Y}$, respectivamente, por 0,$80 ; 0,85 ; 0,90 ; 0,95$ e 0,99 , obtendo os valores de X, as distâncias.

\section{RESULTADOS E DISCUSSÃO}

Nas figuras 2 e 3, observa-se que, quanto maior o número de anos considerados nas análises para o cálculo dos valores dos coeficientes de determinação, menores foram as variações nos valores do coeficiente de determinação $\left(R^{2}\right)$. Os 

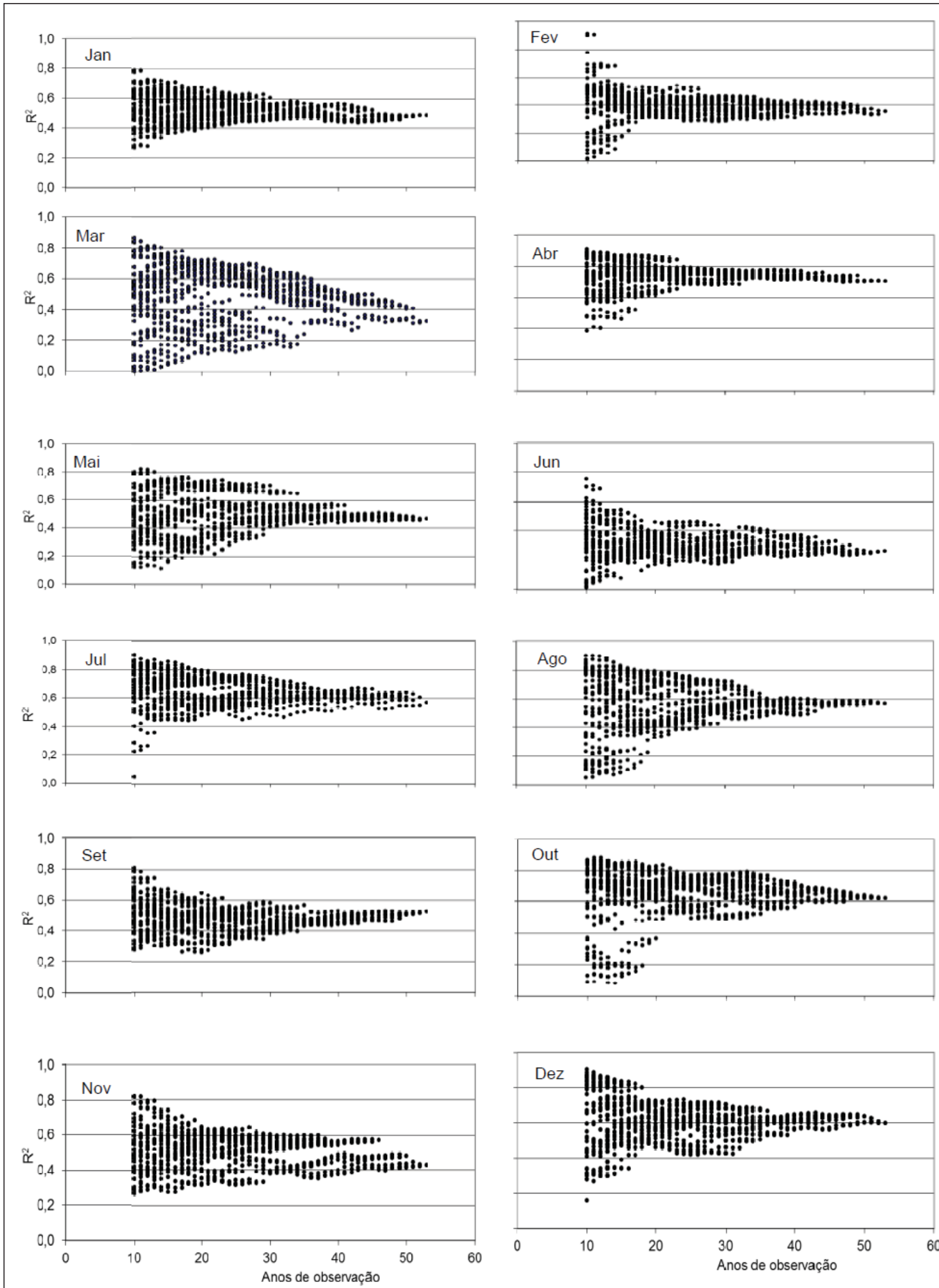

Figura 2 - Relação entre o número de anos de observações e os valores dos coeficientes de determinação ( $\left.\mathrm{R}^{2}\right)$ dos totais mensais de precipitação pluvial, calculados entre as estações de Santa Maria e São Gabriel (RS), período de 1965 a 2008. 

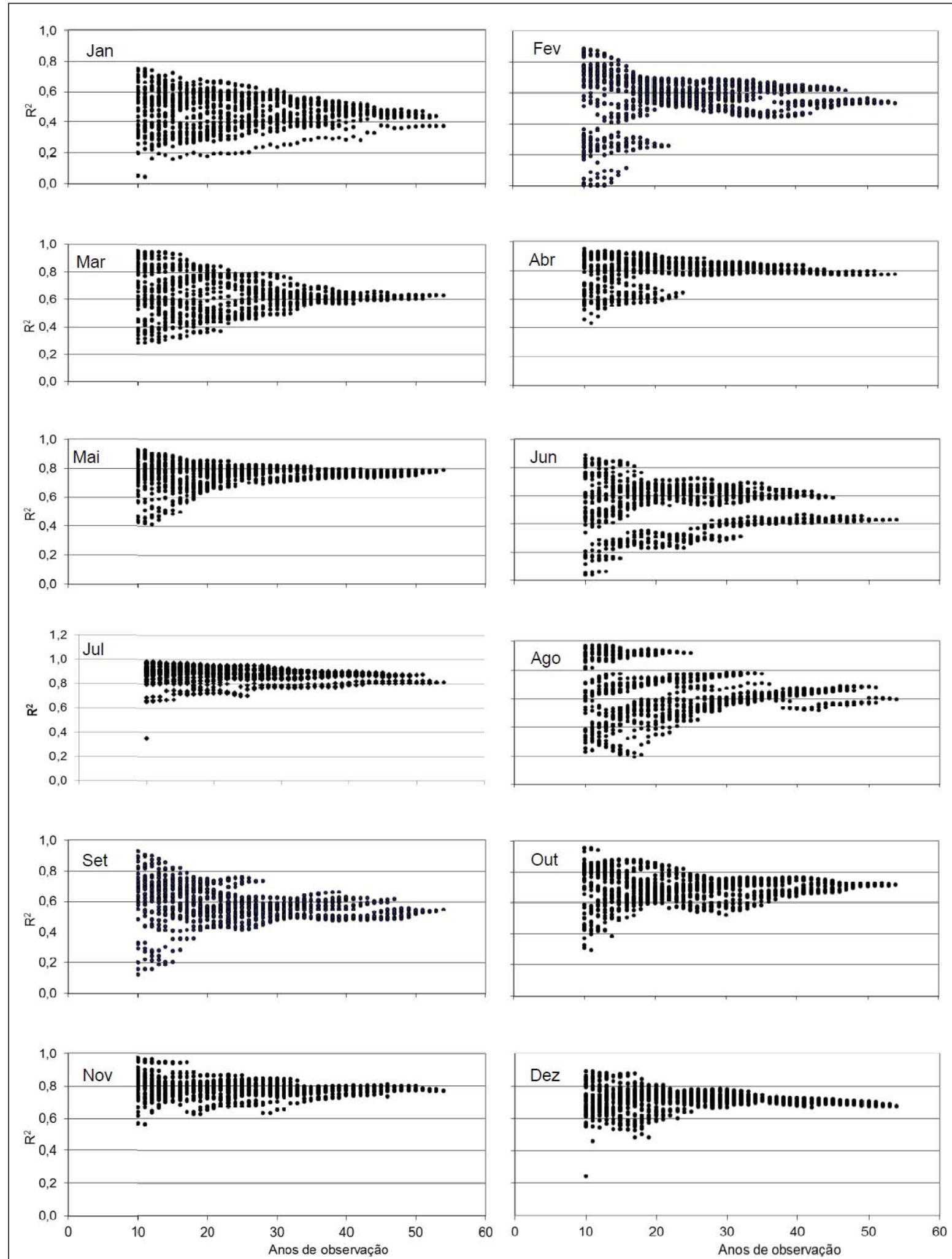

Figura 3 - Relação entre o número de anos de observações e os valores dos coeficientes de determinação $\left(\mathrm{R}^{2}\right)$ dos totais mensais de precipitação pluvial, calculados entre as estações de Santa Maria e Cachoeira do Sul (RS), período de 1965 a 2008. 
decréscimos nas variações dos coeficientes de determinação foram acentuados para os períodos mais curtos de observação, e foram diminuindo à medida que aumentava esse período. A partir de certo tempo, os decréscimos nas variações se tornaram menores, havendo quase estabilidade na amplitude deles. Visualmente, observa-se que a variabilidade dos coeficientes de determinação se torna menor com 25 anos (em fevereiro, abril e dezembro) a 36 anos de observações (em janeiro, julho e novembro). Os valores de $\mathrm{R}^{2}$ estabilizaram em torno de 0,5 , nos meses de maio, junho e dezembro, e nos outros meses em torno de 0,7. Esses valores induzem a indicar o uso de estações com registros de precipitação pluvial de, aproximadamente, 40 anos, como preconiza a Organização Meteorológica Mundial para as regiões subtropicais (CASTILLOS \& SENTIS, 1996). Verifica-se também que, na maioria dos meses, a correlação entre as quantidades de precipitação pluvial ocorridas em Santa Maria e São Gabriel e em Santa Maria e Cachoeira do Sul é baixa, embora a distância entre elas não seja muito elevada.

Os resultados deste trabalho diferem daqueles obtidos por CAMARGO et al. (2005), para o estado de São Paulo, utilizando dados diários de precipitação pluvial. Eles constataram que séries de dados a partir de 20 anos são suficientes para a obtenção de estabilidade nas análises estatísticas. Essas diferenças são devidas, possivelmente, à elevada variabilidade dos dados registrados no estado do Rio Grande do Sul (ÁVILA,1994) e, consequentemente, nas estações pluviométricas localizadas na bacia hidrográfica do Rio Vacacaí (SILVEIRA, 2012). Essa elevada variabilidade se deve, possivelmente, às características das massas de ar que predominam no estado.

$\mathrm{Na}$ figura 4, estão representados os valores de $\mathrm{R}^{2}$ e do erro padrão de estimativa (SEE), em função das distâncias lineares entre a estação pluviométrica de Santa Maria e as outras 12 estações utilizadas. Constatou-se uma elevada variabilidade nos valores de $\mathrm{R}^{2}$ e de SEE. À medida que a distância entre a estação de Santa Maria e as demais estações utilizadas aumentaram, menores foram os valores de $\mathrm{R}^{2}$ e maiores aqueles de SEE. Observou-se que, nos meses de verão, o incremento de SEE aumentou rapidamente com o incremento das distâncias e, nos meses de inverno, mais lentamente. Nos meses de janeiro e fevereiro, ocorreu um grande incremento até, aproximadamente, $5 \mathrm{~km}$ e, após, aumentou lentamente. Entretanto, de março a junho, o maior aumento foi observado até em torno de 10km e, após, o incremento, embora menor, continuou a crescer de forma intensa. A variação dos valores de $R^{2}(Y)$ seguiu funções lineares ( $\mathrm{Y}=\mathrm{a}-\mathrm{bX}$ ) entre as distâncias lineares em km (X), com exceção de dezembro, janeiro e fevereiro, que seguiram funções exponenciais, e aquela dos valores de SEE, funções logarítmicas $[\mathrm{Y}=\mathrm{a}+\ln (\mathrm{x})]$, daqueles obtidos por CAMARGO et al. (2005), que obtiveram funções exponenciais tanto para os valores de $\mathrm{R}^{2}(\mathrm{Y})$ como para aqueles de SEE.

Os limites máximos de distância $(\mathrm{km})$ admitidos para obter concordância de 99, 95, 90, 85 e $80 \%$ entre totais mensais da precipitação pluvial de quaisquer das estações pluviométricas na área da bacia hidrográfica do Rio Vacacaí variaram com os meses do ano, tabela 1. Para os meses de verão, essa distância foi praticamente igual a zero e, para os outros meses do ano, variam de, entre março, 25,0 a $44,4,18,8$ a $33,3,12,5$ a $22,2,6,3$ a 11,1 e 1,3 a 2,2km, respectivamente, para $80 \%$, 85\%, 90\%, 95\% e $99 \%$ de precisão. Esses valores foram menores do que os obtidos no estado de São Paulo para os dados diários (CAMARGO et al., 2005) e um pouco mais elevados daqueles obtidos por HUBBARD (1994) para a região central semi-árida dos EUA. Além dos movimentos convectivos do ar, principalmente nos períodos mais quentes do ano, o fator mais preponderante no condicionamento, tanto pelas semelhanças como pelas diferenças, possivelmente, seja o relevo da região (Figura 1): a altitude e a exposição cardinal.

Pelos resultados obtidos neste trabalho, induz-se que, para a Região da Depressão Central do estado, na representação espacial da precipitação pluvial, é importante utilizar séries de dados de estações pluviométricas com mais de 25 anos de registros como referência e, daquelas com menos de 25 anos de observações, somente como auxiliares.

\section{CONCLUSÃO}

Na bacia do Rio Vacacaí, para ter uma razoável precisão nos totais mensais de precipitação pluvial, são necessários de 25 a 36 anos de observação. Nos meses de verão (dezembro, janeiro e fevereiro), a abrangência das estações pluviométricas da bacia do Rio Vacacaí é menor do que $0,2 \mathrm{~km}$. Nos outros meses, as distâncias variam entre 1,3km (99\% de precisão) até 44,4km (80\% de precisão).

\section{AGRADECIMENTOS}

Ao Conselho Nacional de Desenvolvimento Científico e Tecnológico (CNPq), pelo financiamento e bolsas concedidas. 


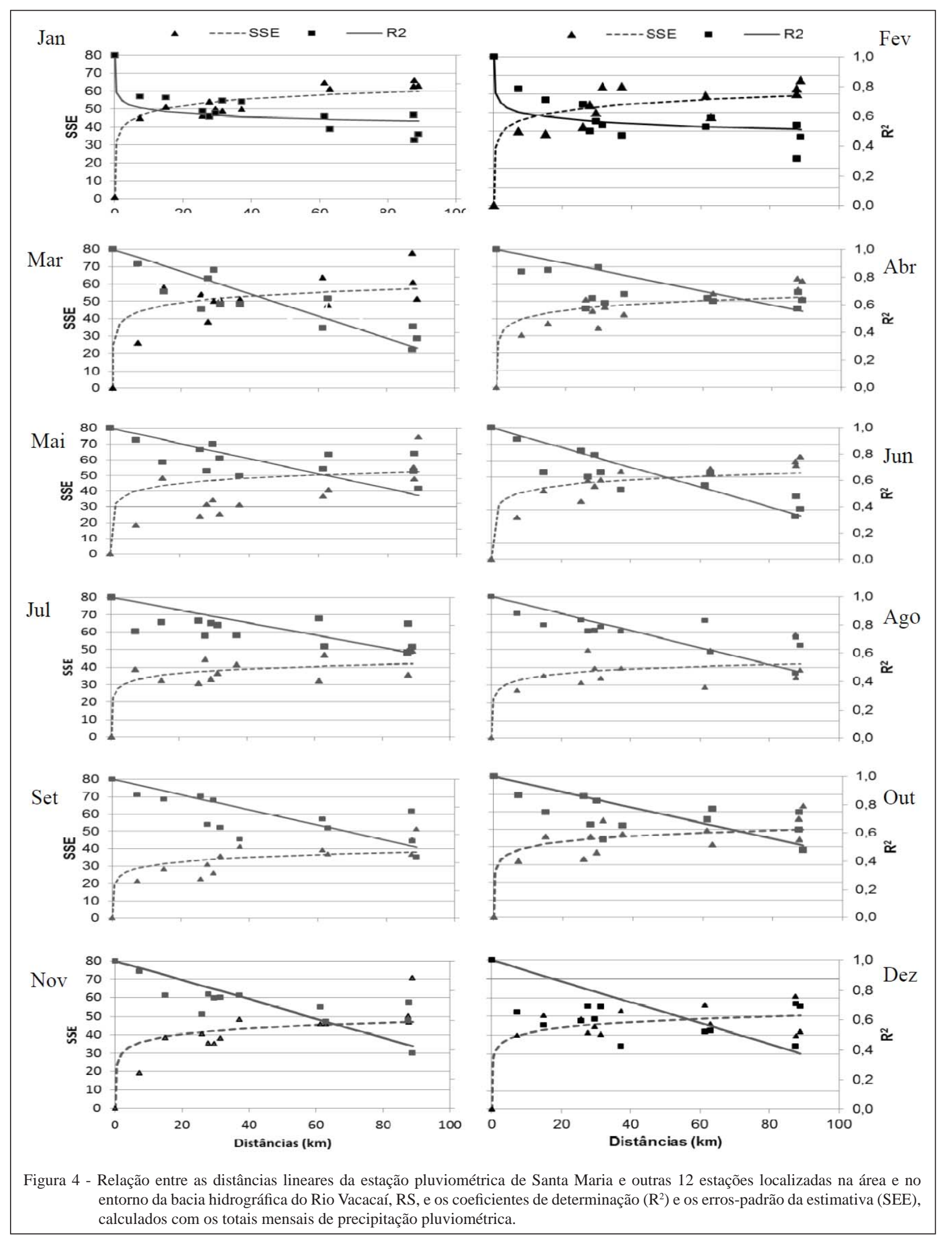

Ciência Rural, v.43, n.12, dez, 2013. 
Tabela 1 - Abrangência (distância em km) entre estações pluviométricas da bacia do Rio Vacacaí com alguns níveis de precisão (concordância).

\begin{tabular}{|c|c|c|c|c|c|c|c|c|c|c|c|c|}
\hline Precisão (\%) & Jan & Fev & Mar & Abr & Mai & Jun & Jul & Ago & Set & Out & Nov & Dez \\
\hline 99 & 0,0 & 0,0 & 1,3 & 2,0 & 1,7 & 1,3 & 2,2 & 1,7 & 1,8 & 1,8 & 1,5 & 0,0 \\
\hline 95 & 0,0 & 0,0 & 6,3 & 10,0 & 8,3 & 6,7 & 11,1 & 8,3 & 9,1 & 9,1 & 7,7 & 0,0 \\
\hline 90 & 0,0 & 0,0 & 12,5 & 20,0 & 16,7 & 13,3 & 22,2 & 16,7 & 18,2 & 18,2 & 15,4 & 0,0 \\
\hline 85 & 0,1 & 0,0 & 18,8 & 30,0 & 25,0 & 20,0 & 33,3 & 25,0 & 27,3 & 27,3 & 23,1 & 0,0 \\
\hline 80 & 0,1 & 0,0 & 25,0 & 40,0 & 33,3 & 26,7 & 44,4 & 33,3 & 36,4 & 36,4 & 30,8 & 0,2 \\
\hline
\end{tabular}

\section{REFERÊNCIAS}

ANA (AGÊNCIA NACIONAL DAS ÁGUAS). Hidroweb: sistema de informações hidrológicas. Disponível em: <http:// hidroweb.ana.gov.br>. Acesso em: out. 2010.

ÁVILA, A.M.H. Regime de precipitação pluvial no estado do Rio Grande do Sul com base em séries de longo prazo. 1994. 75f. Dissertação (Mestrado em Agronomia) - Programa de Pósgraduação em Agronomia, Faculdade de Agronomia, Universidade Federal do Rio Grande do Sul, Porto Alegre, RS.

BERTONI, J.C.; TUCCI, C.E.M. Precipitação. In: TUCCI, C.E.M. Hidrologia: ciência e aplicação, 2001. Porto Alegre: UFRGS, 2002. p.176-241.

CASTILLO, F.E.; SENTIS, F.C. Agrometeorologia. Madrid: Mundi-Prensa Libros, 1996. 517p.

CAMARGO, M.B.P.; HUBBARD, K.G. Spatial and temporal variability of daily weather variables in sub-humid and semiarid portions of the United States high plains. Agricultural and Forest Meteorology, Amsterdam, v.93, p.141-148, 1999. Disponível em: <http://www.sciencedirect.com/science/article/ pii/S0168192398001221>. Acesso em: 20 nov. 2012. doi:10.1016/ S0168-1923(98)00122-1.

CAMARGO, M.B.P. de et al. Variabilidade espacial e temporal de dados termopluviométricos diários da rede de estações agrometeorológicas do Instituto Agronômico (IAC). Bragantia, Campinas, v.64, n.3, p.475-485, 2005. Disponível em: <http:/ www.scielo.br/scielo.php? script=sci_pdf\&pid=S0006$87052005000300017 \& \operatorname{lng}=p t \& n r m=i s o \& t \operatorname{lng}=p t>$. Acesso em: 20 nov. 2012. doi: 10.1590/S0006-87052005000300018.
MELLAART, E.A.R. Small-scale spatial rain distribution: the effect of temporal and spatial rain distribution on drought and crop yield at village level. Nelspruit: Institute for Soil, Climate and Water, 1999. 18p.

NORDENSON, T.J. Preparation de cartes coordonees dês precipitations, de l'ecoulement et de l'evaporation. Geneve: Organization Meteorologique Mondiale, 1971. 36p. (Rapport n.6).

OMETTO, J.C. Bioclimatologia vegetal. São Paulo: Agronômica Ceres, 1981. 425p.

PRÓ-GUAÍBA. Região hidrográfica do Guaíba: nove bacias e suas características. Disponível em: <http://www.proguaiba. rs.gov.br/bacias.htm>. Acesso: 29 dez. 2011.

RS - Secretaria da Saúde e Meio Ambiente (SSMA). Portaria n.05/89 de 16 de Março de 1989. Aprova a Norma Técnica que dispõe sobre critérios e efluentes líquidos. Diário Oficial, Porto Alegre, 29 de março de 1989.

SEMA (SECRETÁRIA ESTADUAL DO MEIO AMBIENTE). Sistema estadual de recursos hídricos, 2009. Disponível em: <http://www.sema.rs.gov.br/sema/jsp/rechidro.jsp>. Acesso: 20 jul. 2011.

SIEGEL, S. Estatística não paramétrica. São Paulo: Makron Books do Brasil, 1975. 349p.

SILVEIRA, P. da C. Precipitação pluviométrica na bacia hidrográfica do Rio Vacacaí, RS. 2012. 54f. Dissertação (Mestrado em Geografia) - Programa de Pós-graduação em Geografia e Geociência, UFSM, Santa Maria, RS. 